\title{
Thoracoscopic lobectomy: Report on safety, discharge independence, pain, and chemotherapy tolerance
}

\author{
Daniel G. Nicastri, MD, ${ }^{\text {a }}$ Juan P. Wisnivesky, MD, MPH, ${ }^{\mathrm{b}}$ Virginia R. Litle, MD, ${ }^{d}$ Jaime Yun, MD, ${ }^{d}$ Cynthia Chin, MD, ${ }^{d}$
} Francine R. Dembitzer, MD, ${ }^{c, d}$ and Scott J. Swanson, MD ${ }^{d}$

Earn CME credits at http:// cme.ctsnetjournals.org
From the Departments of Surgery, ${ }^{\text {a }}$ Medicine, ${ }^{\mathrm{b}}$ and Pathology, ${ }^{\mathrm{c}}$ and Division of Thoracic Surgery, ${ }^{\mathrm{d}}$ Mount Sinai Medical Center, New York, NY.

Received for publication March 7, 2007; revisions received Sept 10, 2007; accepted for publication Sept 24, 2007.

Address for reprints: Scott Swanson, MD, Mount Sinai Medical Center, 1190 Fifth Avenue, New York, NY 10029 (E-mail: scott.swanson@mountsinai.org).

J Thorac Cardiovasc Surg 2008;135:642-7 $0022-5223 / \$ 34.00$

Copyright $(\subset) 2008$ by The American Association for Thoracic Surgery

doi:10.1016/j.jtcvs.2007.09.014
Objective: Controversies regarding the safety, morbidity, and mortality of thoracoscopic lobectomy have prevented the widespread acceptance of the procedure. This series analyzed the safety, pain, analgesic use, and discharge disposition in patients who underwent thoracoscopic lobectomy and segmentectomy at a single institution.

Methods: We collected data from 153 consecutive patients who underwent thorascopic (video-assisted thoracic surgery) lobectomy and assessed the perioperative outcomes, postoperative pain, and chemotherapy course. A total of 111 of 127 patients with lung cancer had stage I non-small cell lung cancer. The operative technique required 2 ports and an access incision $(5-8 \mathrm{~cm})$, individual hilar ligation, and lymph node dissection performed without rib-spreading devices.

Results: There were 9 major complications (6\%), including 1 perioperative death $(0.7 \%)$. Conversion to thoracotomy occurred in 14 patients $(9.2 \%)$. Blood transfusion was required in 11 patients $(7 \%)$. The median chest tube time was 3 days, and the length of hospital stay was 4 days; $94.4 \%$ of patients went home at the time of discharge, and $5.6 \%$ of patients required a rehabilitation facility. At a median postsurgical follow-up time of 2 weeks, the mean postoperative pain score was $0.6(0-3), 73 \%$ of patients did not use narcotics for pain control, and $47 \%$ of patients did not use any pain medication. Of patients receiving chemotherapy $(\mathrm{N}=26), 73 \%$ completed a full course on schedule and $85 \%$ received all intended cycles.

Conclusion: Thoracoscopic (video-assisted thoracic surgery) lobectomy can be performed safely. Discharge independence and low pain estimates in the early postoperative period suggest that this approach may be beneficial. Furthermore, there is a trend toward improved tolerance of chemotherapy.

$\mathrm{T}$ horacoscopic (video-assisted thoracic surgery [VATS]) lobectomy has been used in the treatment of lung cancer since the early 1990s and has become an accepted method of lobectomy. ${ }^{1}$ Several small VATS series reported that VATS lobectomy is technically feasible and safe. In our series, the data confirm results obtained in other retrospective series with regard to low mortality and morbidity. We provide additional data about postoperative pain control, discharge disposition, and tolerance of chemotherapy.

\section{Materials and Methods}

\section{Patient Selection, Preoperative Studies, Procedure, Perioperative Care}

The study population included patients with suspected early-stage (stages IA or IB, clinical stage I) lung cancer and consisted of 153 patients who underwent an attempted thoracoscopic lobectomy or segmentectomy in a single tertiary care hospital in New York City between January of 2002 and April of 2006. A total of 127 of these 153 patients had lung cancer. Our lung cancer cohort during this time period included 199 patients; 37 of these patients were not eligible for VATS lobectomy because of tumor size or location and underwent lobectomy or segmentectomy by thoracotomy. Of the 162 remaining patients eligible for VATS, a total of 92 underwent mediastinoscopy. Pathologically positive nodes were found in 35 patients who 


\section{Abbreviations and Acronyms}

NSCLC $=$ non-small cell lung cancer

VATS $=$ video-assisted thoracic surgery

then underwent induction chemotherapy/radiation and were excluded from the VATS study group. An additional 57 of the 127 VATS lung cancer cohort had negative mediastinoscopies.

Patients were included in the VATS cohort study data if the preoperative surgical goal was thoracoscopic lobectomy regardless of whether the surgery was completed thoracoscopically. However, if our intention was to perform an exploratory thoracoscopy to rule out pleural disease, and a subsequent resection by thoracotomy was performed, the case was excluded from the VATS series.

All patients with stages I and II lung cancer who did not have contraindications to the procedure were eligible for VATS lobectomy. The VATS study exclusion criteria were the standard absolute contraindications to VATS lobectomy and included the presence of T3 or T4 tumors, N3 disease, or inability to achieve single-lung ventilation. Relative contraindications included central hilar tumors, tumors that were visible on bronchoscopy or necessitated sleeve resection, bulky mediastinal or hilar lymphadenopathy, and a history of neoadjuvant chemotherapy or radiation.

If a conversion was necessary, patients remained in this cohort on the basis of the principle of "intention to treat." All cases were discussed with the operating surgeon. Operative notes were reviewed, and the goals for the surgical procedures were discussed. Thus, we were able to distinguish patients who underwent thoracoscopy to rule out pleural metastases from those in whom conversions were performed. The operative goal for all patients with presumed non-small cell lung cancer (NSCLC) stages I and II was a thoracoscopic surgical procedure. The reasons for conversions are listed in the "Results" section.

Preoperative studies for VATS lobectomy included chest radiograph, computed tomography scan, bronchoscopy, pulmonary function studies, positron emission tomography scan, and other modalities for metastatic workup when necessary. The type of surgery performed was individualized to the patient and the tumor. Most commonly, we performed lobectomies. However, in patients with poor pulmonary function and smaller tumors, anatomic segmentectomies were performed. A summary of resections performed by anatomic location is shown in Table 1 .

TABLE 1. Resections by anatomic location $(n=153)$

\begin{tabular}{lcr}
\hline \multicolumn{1}{c}{ Location } & Quantity & Percent \\
\hline Right upper lobectomy & 56 & $36.6 \%$ \\
Right middle lobectomy & 11 & $7.2 \%$ \\
Right lower lobectomy & 31 & $20.3 \%$ \\
Left upper lobectomy & 26 & $17.0 \%$ \\
Left lower lobectomy & 19 & $12.4 \%$ \\
Lingulectomy & 3 & $2.0 \%$ \\
Superior segmentectomy left & 2 & $1.3 \%$ \\
Superior segmentectomy right & 3 & $2.0 \%$ \\
Bilobectomy & 2 & $1.3 \%$ \\
Total & 153 & $100.0 \%$ \\
\hline
\end{tabular}

We performed bronchoscopy on all patients after intubation with a double-lumen tube and before repositioning. Mediastinoscopy was performed on patients with lymph node enlargement greater than $1 \mathrm{~cm}$ or positive lymph-node findings on positron emission tomography scan. We prefer 3 incisions: an inferior camera port, a posterior working port, and an anterior access/utility incision. The avoidance of rib spreading is mandatory or we consider the case a conversion. The first port is the camera port, and it is usually at the seventh or eighth intercostal space. Ideally, this port should provide views of the anterior and posterior hila and should align with the major fissure. We almost exclusively use a 30-degree thoracoscope. The anterior port is placed directly over the hilum. It is usually created anterior to the latissimus dorsi in the fourth intercostal space for upper lobectomies and in the fifth intercostal space for middle/lower lobectomies. Dissection of both the hilum and fissure is performed through this port. This initial incision is 1 to $2 \mathrm{~cm}$, and it is extended to 5 to $8 \mathrm{~cm}$ in length only if we proceed with the VATS lobectomy. The third port is usually in the fifth or sixth intercostal space, either inferior, or posterior, to the scapular tip. This port usually serves as the lung retraction port. Individual hilar ligation is performed, and lymph nodes are dissected.

All patients underwent lymph node dissection. We dissected lymph nodes 2, 4, and 7 to 12 (or hilar) for right-sided tumors, lymph node stations 5 to 12 for left-sided tumors, and $4 \mathrm{R}, 4 \mathrm{~L}$, and 7 for cervical mediastinoscopies. Typically for T1 tumors, we did not perform routine mediastinoscopy unless the preoperative computed tomography scan demonstrated lymphadenopathy greater than $1 \mathrm{~cm}$. We routinely performed mediastinoscopies in patients with positron emission tomography-positive mediastinal adenopathy (standard uptake value $>3$ ) or T2 or greater-sized tumors. After surgery, all patients recovered in the postoperative anesthesia care unit and were transferred subsequently to a monitored care setting.

\section{Chemotherapy}

Postoperatively, patients who were eligible received adjuvant chemotherapy according to the Cancer and Leukemia Group B 9633 North American Intergroup JBR.10 (JBR.10) protocols, namely, 4 cycles of paclitaxel and carboplatin for 12 weeks or 4 cycles of cisplatin and vinorelbine for 16 weeks, respectively. Most patients were treated with the Cancer and Leukemia Group B protocol; 1 patient received the JBR.10 protocol. ${ }^{2,3}$ The adjuvant chemotherapy data were collected from patient charts and interviews, and oncologist interviews. Not all patients received chemotherapy at our institution, but follow-up was complete and all patients were included.

\section{Postoperative Follow-up Care}

Data were obtained and analyzed retrospectively. During postsurgical visits, patients were asked by nurse practitioners to grade their level of pain responses in a questionnaire format. Patients evaluated their pain levels on a scale of 0 to $3(0=$ no pain, $1=$ mild pain, $2=$ moderate pain, $3=$ severe pain). In addition, patients categorized their use of medications for pain relief as none, nonsteroidal anti-inflammatory agents, opiates, or both. The postoperative interval of their visit was also recorded. Patients were contacted with approval of the institutional review board for chemotherapy, recurrence, and survival data. Individual consent was waived. 


\section{Complications}

Major complications were considered mortality, respiratory failure, empyema, atrial fibrillation requiring cardioversion or anticoagulation, and reoperation. Minor complications were considered prolonged air leak, atrial fibrillation not requiring cardioversion and anticoagulation, pneumonia without respiratory failure, deep vein thrombosis, urinary tract infection, and cellulitis from intravenous catheter. These data were collected retrospectively on the basis of chart review, postoperative follow-up visits, discharge summaries, and patient interviews.

\section{Statistical Analysis}

Medians and ranges are presented for non-normal data. We used the Kaplan-Meier method to estimate disease-free survivals of patients with stage I NSCLC who underwent VATS lobectomy. To estimate disease-free survival, we treated deaths from causes other than lung cancer as censored observations.

\section{Results}

From January of 2002 to April of 2006, 153 patients underwent thoracoscopic lobectomies at our institution. The series comprised 67 men and 86 women. The patient characteristics are shown in Table 2. Of 153 VATS anatomic resections performed, the pathologic diagnosis was NSCLC in 127 patients (83\%) (Table 3$)$. The most common NSCLC was adenocarcinoma; other surgical indications included carcinoid tumors, metastases, and suspicious lesions, usually granuloma or hamartoma (Table 4). A number of patients with advanced tumors underwent VATS surgery, which are grouped into the following categories: oligometastatic disease for which aggressive treatment is recommended $(n=2)$; multiple nodules but with negative lymph nodes, thereby treating as synchronous rather than metastatic lesions $(n=2)$; falsenegative readings on lymph nodes taken during mediastinoscopy or an occult metastatic lymph node $(\mathrm{n}=2)$; and a significant interval of 11 years between the initial tumor and the more recent tumor mass $(n=1)$.

\section{Hospital Course}

The median length of time for chest tube removal was 3 days; the median length of hospital stay was 4 days. Some 52 of 153 patients (34\%) were discharged on or before postoperative day $3 ; 96$ of 144 patients $(66.6 \%)$ were discharged

\section{TABLE 2. Group characteristics}

\begin{tabular}{lcc}
\hline Characteristic & Total/range & Median \\
\hline Age & $36-86$ & 67 \\
Male & 67 & \\
Female & 86 & \\
FEV $\%$ predicted & $29-153$ & 88 \\
ECOG scores & $0-2$ & 0 \\
Tumor $(\mathrm{cm})$ & $0.6-6.0$ & 2.2
\end{tabular}

$F E V_{1}$, Forced expiratory volume in 1 second; ECOG, Eastern Cooperative Oncology Group.
TABLE 3. Non-small cell lung cancer stages ( $\mathbf{n}=127$ )

\begin{tabular}{lrr}
\hline Stage & No. & Percent \\
\hline 1A & 75 & $59.1 \%$ \\
1B & 36 & $28.3 \%$ \\
$2 A$ & 5 & $3.9 \%$ \\
2B & 4 & $3.1 \%$ \\
3A & 2 & $1.6 \%$ \\
$3 B$ & 2 & $1.6 \%$ \\
4 & 3 & $2.4 \%$ \\
Total & 127 & $100.0 \%$ \\
\hline
\end{tabular}

without any nursing assistance at home; 40 of 144 patients (27.8\%) were discharged with home assistance; 8 of 144 patients $(5.6 \%)$ were discharged to a rehabilitation facility. Of the 40 patients discharged with home assistance, 37 $(92.5 \%)$ received brief nursing visits (eg, Visiting Nurses Association of America) and $3(7.5 \%)$ required home assistance for several hours per day.

\section{Mortality and Morbidity}

There were no intraoperative deaths. There was 1 postoperative death $(0.7 \%)$ secondary to respiratory failure from aspiration pneumonia. The most common complications were atrial fibrillation in 16 of 153 patients (10.5\%) and prolonged air leak more than 7 days in 12 of 153 patients (7.8\%). Most patients with atrial fibrillation had a short course, with a majority requiring chemical cardioversion with amiodarone. Two patients underwent electrical cardioversion, and 2 patients were anticoagulated. Some patients experienced more than 1 complication. Other complications are noted in Table 5.

\section{Conversion to Thoracotomy}

We considered conversion to include a standard thoracotomy or a minithoracotomy with any degree of rib spreading. There were no conversions resulting from the inability to tolerate single-lung ventilation. In 14 cases $(9.2 \%)$, the procedure was converted to either a standard thoracotomy or a minithoracotomy for the following reasons: controlled bleeding of a pulmonary artery branch $(n=5)$, cancer involvement of the hilar structures $(n=6)$, or other anatomic considerations

TABLE 4. Histopathologic diagnosis $(n=153)$

\begin{tabular}{lrr}
\hline \multicolumn{1}{c}{ Type } & No. & Percent \\
\hline Adenocarcinoma & 95 & $62.1 \%$ \\
Squamous cell & 21 & $13.7 \%$ \\
Other NSCLC & 11 & $7.8 \%$ \\
Carcinoid & 9 & $5.9 \%$ \\
Metastases & 7 & $4.6 \%$ \\
Other & 9 & $5.9 \%$ \\
Total & 153 & $100.0 \%$ \\
\hline
\end{tabular}

NSCLC, Non-small cell lung cancer. 
TABLE 5. Complications after surgery $(n=153)$

\begin{tabular}{lrr}
\hline \multicolumn{1}{c}{ Complication } & No. & Percent \\
\hline Atrial fibrillation & 16 & $10.5 \%$ \\
Air leak & 12 & $7.8 \%$ \\
Respiratory failure & 3 & $2.0 \%$ \\
Empyema & 2 & $1.3 \%$ \\
Pneumonia & 3 & $2.0 \%$ \\
Deep vein thrombosis & 1 & $0.7 \%$ \\
Other minor & 5 & $3.3 \%$ \\
Total & 42 & $27.5 \%$ \\
\hline
\end{tabular}

$(\mathrm{n}=3)$. Of the 5 patients with pulmonary arterial bleeding, control was achieved with a spongestick before conversion to a standard thoracotomy in 4 patients and minithoracotomy in 1 patient. Two of 5 patients with pulmonary artery bleeding received a blood transfusion. Overall, blood transfusions were administered to 11 patients $(7.2 \%)$, most often secondary to significant adhesions or coagulopathy.

\section{Postoperative Pain}

At the first postoperative follow-up visit at a median time of 2 weeks (mean 3.1 weeks) postsurgery, patients were asked to assess their level of pain on a scale of 0 to 3 ( $0=$ in no pain; $1=$ in mild pain; $2=$ in moderate pain; $3=$ in severe pain) The mean pain score based on this scale was 0.6 (median 0; range $0-3)$. Patients $(\mathrm{N}=145)$ also reported the medications they were using for pain relief. At this median follow-up period of 2 weeks, $47 \%$ of patients were not using any pain medication, $26 \%$ were taking NSAIDs either as needed or standing order, and $27 \%$ reported some narcotic use, with most, $22 \%$, taking opiates as needed.

\section{Oncologic Results}

Of 127 patients with NSCLC who underwent thoracoscopic lobectomies, 57 (44.9\%) had cervical mediastinoscopies, all of which were negative. Seventy patients did not undergo mediastinoscopy. The mean number of lymph node stations sampled from the patients with NSCLC was 4.8 overall (median, 5.0), including mediastinoscopy and lymph node dissection. Eleven patients were diagnosed with positive lymph nodes $(11 / 127 ; 8.7 \%)$ after the thoracoscopic lymph node dissection; 6 of these patients had negative mediastinoscopies.

\section{Chemotherapy}

Of the 26 patients who received chemotherapy, 19 (73\%) received their full planned dose on schedule and $3(12 \%)$ were administered the full course of planned doses of chemotherapy with a delay in 1 or more cycles. Twenty-two patients $(85 \%)$ received all intended cycles with or without delay. Reasons for dose delay or dose reduction in our series were neutropenia $(\mathrm{n}=2)$, gastrointestinal symptoms $(\mathrm{n}=2)$, unknown $(\mathrm{n}=3)$, and a combination of symptoms $(\mathrm{n}=1)$.

\section{Survival}

Follow-up was complete for all patients with lung cancer. The median follow-up time was 14 months (mean: 16 months; range: 1-40 months). Fourteen patients had recurrences: 1 of 127 patients $(0.8 \%)$ had a locoregional recurrence at 26 months; 7 of 127 patients $(5.5 \%)$ had distant recurrences; and 6 of 127 patients $(4.7 \%)$ had both locoregional and distant recurrences. Our rate of isolated locoregional recurrence is low, but because this is a small group with a short follow-up period, no definite conclusions may be drawn. Figure 1 summarizes disease-free survival for all patients with NSCLC (follow-up data are for 124 of 127 patients; 3 patients had 2 separate lobectomies for metachronous lesions, and survival data are assessed in each case for only 1 of the 2 surgical procedures). Figure 2 summarizes all patients with stage I NSCLC.

\section{Discussion}

VATS lobectomy should no longer be considered a new technology; these results and others demonstrate that it has become the preferred approach at many medical centers. Once the technique is mastered, this surgical procedure can be performed safely with excellent oncologic and perioperative outcomes. Discharge independence and low pain estimates in the early postoperative period suggest this approach may be beneficial.

One criticism of this surgery is the risk of uncontrolled bleeding. Although we recognize that this risk exists, it seems to be minimal and has not been reported in many large series. Should pulmonary arterial injury occur during a VATS procedure, control can easily be achieved with a spongestick, and the surgeon can convert to a thoracotomy. Furthermore, the safety and efficacy of VATS lobectomy have been demonstrated in several small series, some of these prospective

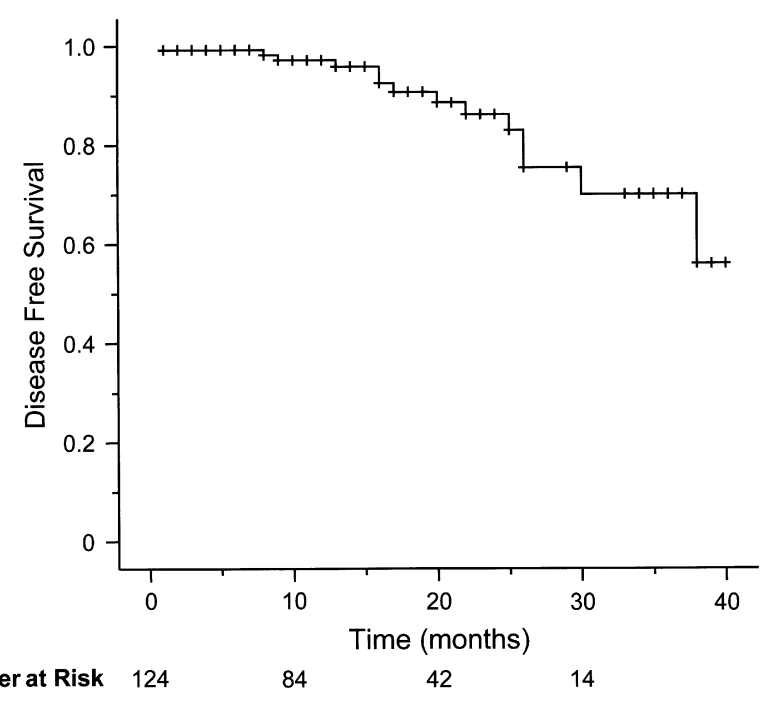

Figure 1. Disease-free survival in all patients with NSCLC. 


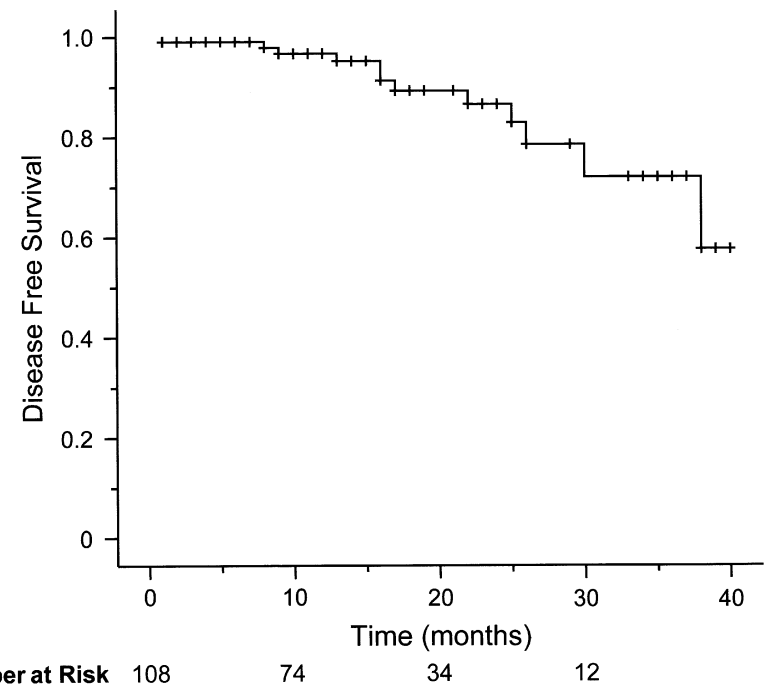

Figure 2. Disease-free survival in patients with stage I NSCLC.

and randomized. ${ }^{4-7}$ The comparative safety profiles between VATS and thoracotomy are similar. Recently, McKenna and colleagues ${ }^{8}$ published a large series with excellent perioperative outcomes. Perioperative morbidity and mortality with VATS lobectomy compare favorably to those of large series of thoracotomy lobectomies. In the largest series of patients undergoing lobectomy predominantly by thoracotomy, morbidity ranged from $28 \%$ to $38 \%$ and mortality ranged from $1.2 \%$ to $2.9 \% .^{9-11}$ Other VATS lobectomy series have had morbidity rates ranging from $9 \%$ to $19 \%$, with perioperative mortality ranging from $0.8 \%$ to $1.8 \% .^{8,12-15}$

\section{Oncologic Results}

VATS lobectomy is oncologically the same surgical procedure as a lobectomy through a thoracotomy; both use anatomic resection, individual hilar ligation, and lymph node sampling or dissection. Reports indicate that the number of dissected lymph nodes is similar between VATS lobectomy and thoracotomy. ${ }^{16-18}$ Uniform lymph node counting procedures at this institution were established subsequent to our review so we cannot report the number of lymph nodes dissected per VATS case here. Five-year survivals are comparable and in some cases seemingly better. Walker and colleagues $^{12}$ reported a 4-year survival of $78 \%$ in 117 patients with stage I lung cancer, whereas other studies have reported 5-year survivals as high as $97 \% .{ }^{19}$ This compares with published 5-year survivals between $61 \%$ and $82 \%$ for patients with stage I surgical lung cancer who undergo thoracotomy, although admittedly a direct comparison would be unfair because VATS data are not based on randomized prospective controlled studies and may be related to patient selection. ${ }^{20,21}$

\section{Postoperative Pain, Length of Stay, and Discharge Disposition}

The greatest advantage of a VATS lobectomy may be an improvement in patients' perioperative quality of life. The pain associated with this surgery is probably less than that experienced by patients who undergo thoracotomy and may in part be explained by decreased inflammation. ${ }^{22}$ Other series have suggested that this pain is significantly less in the early postoperative period..$^{4,5,7}$ In a case-control study, Demmy and colleagues ${ }^{23}$ reported similar discharge data after VATS lobectomies and found that significantly more patients who underwent thoracotomy required skilled nursing facilities after surgery. Thus, the data suggest that these patients have short hospital stays, are discharged without home assistance, and have low opiate requirements.

\section{Chemotherapy}

Although we obtained some preliminary data on a small group of patients who underwent both VATS lobectomy and adjuvant chemotherapy, we were not able to make any definitive comparisons with regard to tolerance of chemotherapy compared with open lobectomy groups. There are few data in the literature about how soon after surgery a patient begins adjuvant chemotherapy, although most trials seem to start after a postsurgical interval of 6 weeks. Another recent study reported that patients who underwent VATS had significantly less dose delay and dose reduction, but the interval to delivery of chemotherapy was not significant. ${ }^{24}$ In comparison, the Cancer and Leukemia Group B trial 9633 reported that $55 \%$ of patients received full-dose chemotherapy and the Intergroup JBR.10 trial reported that $45 \%$ of patients had at least 1 dose delay. ${ }^{2,3}$ Approximately $34 \%$ of patients in the Adjuvant Lung Project Italy series chemotherapy wing received all scheduled doses without adjustment or delay; $69 \%$ completed their treatments with or without adjustments or delay. ${ }^{25}$ It is conceivable that patients who undergo VATS may have a quicker recovery and in general more strength to tolerate chemotherapy. There are theoretic survival benefits to starting chemotherapy immediately after surgery because the body's tumor burden should be lowest, and tumor growth fastest, at this time. Thus, chemotherapy administered immediately postsurgery would be most effective, assuming that wound healing is adequate.

\section{Conclusions}

Our data demonstrate that thoracoscopic lobectomy is a safe procedure with low pain estimates and excellent discharge independence in the early postoperative period. A multiinstitutional prospective series is warranted to answer the question of how thoracoscopic lobectomy compares with open lobectomy in oncologic efficacy, morbidity, and overall potential advantages of this approach, including the timing of postoperative chemotherapy and chemotherapy tolerance. 
We thank Lisa McCaskill, MSW, and Harry Gluck, MD.

\section{References}

1. Ettinger DS, Bepler G, Bueno R, et al. National Comprehensive Cancer Network (NCCN): Non-small cell lung cancer clinical practice guidelines in oncology. J Natl Compr Canc Netw. 2004;2:94-124.

2. Strauss GM, Herndon J, Maddaus MA, Johnstone DW, Johnson EA, Watson DM, et al. Randomized clinical trial of adjuvant chemotherapy with paclitaxel and carboplatin following resection in stage 1B nonsmall-cell lung cancer (NSCLC): Report of Cancer and Leukemia Group B (CALGB) Protocol 9633. J Clin Oncol. 2004;22(Suppl 14):621s (abstract).

3. Winton T, Livingston R, Johnson D, Rigas J, Johnston M, Butts C, et al. Vinorelbine plus cisplatin vs. observation in resected non-small-cell lung cancer. $N$ Engl J Med. 2005;352:2589-97.

4. Nomori H, Horio H, Naruke T, Suemasu K. What is the advantage of a thoracoscopic lobectomy over a limited thoracotomy procedure for lung cancer surgery? Ann Thorac Surg. 2001;72:879-84.

5. Sugiura H, Morikawa T, Kaji M, Sasamura Y, Kondo S, Katoh H. Longterm benefits for the quality of life after video-assisted thoracoscopic lobectomy in patients with lung cancer. Surg Laparosc Endosc Percutan Tech. 1999;9:403-8.

6. Kirby TJ, Mack MJ, Landreneau RJ, Rice TW. Lobectomy-videoassisted thoracic surgery versus muscle-sparing thoracotomy. A randomized trial. J Thorac Cardiovasc Surg. 1995;109:997-1001.

7. Ohbuchi T, Morikawa T, Takeuchi E, Kato H. Lobectomy: videoassisted thoracic surgery versus posterolateral thoracotomy. Jpn J Thorac Cardiovasc Surg. 1998;46:519-22.

8. McKenna RJ Jr, Huock W, Fuller CB. Video-assisted thoracic surgery lobectomy: experience with 1,100 cases. Ann Thorac Surg. 2006;8: 421-5.

9. Allen MS, Darling GE, Pechet TT, Mitchell JD, Herndon JE, Landreneau RJ, et al. Morbidity and mortality of major pulmonary resections in patients with early-stage lung cancer: initial results of the randomized, prospective ACOSOG Z0030 trial. Ann Thorac Surg. 2006; 81:1013-9.

10. Deslauriers J, Ginsberg RJ, Piantadosi S, Fournier B. Prospective assessment of 30-day operative morbidity for surgical resections in lung cancer. Chest. 1994;106(6 Suppl):329S-30.

11. Wada H, Nakamura T, Nakamoto K, Maeda M, Watanabe Y. Thirty-day operative mortality for thoracotomy in lung cancer. $J$ Thoracic Cardiovasc Surg. 1998;115:70-3.

12. Walker WS, Codispoti M, Soon SY, Stamenkovic S, Carnochan F, Pugh G. Long-term outcomes following VATS lobectomy for non-small cell bronchogenic carcinoma. Eur J Cardiothorac Surg. 2003;23: 397-402.

13. Kaseda S, Aoki T, Hangai N. Video-assisted thoracic surgery (VATS) lobectomy: the Japanese experience. Semin Thorac Cardiovasc Surg. 1998;10:300-4.

14. Solaini L, Prusciano F, Bagioni P, Di FF, Basilio PD. Video-assisted thoracic surgery major pulmonary resections. Present experience. Eur J Cardiothorac Surg. 2001;20:437-42.

15. Ohtsuka T, Nomori H, Horio H, Naruke T, Suemasu K. Is major pulmonary resection by video-assisted thoracic surgery an adequate procedure in clinical stage I lung cancer? Chest. 2004;125:1742-6.

16. Kondo T, Sagawa M, Tanita T, Sato M, Ono S, Matsumura Y, et al. Is complete systematic nodal dissection by thoracoscopic surgery possible? A prospective trial of video-assisted lobectomy for cancer of the right lung. J Thorac Cardiovasc Surg. 1998;116:651-2.

17. Kaseda S, Hangai N, Yamamoto S, Kitano M. Lobectomy with extended lymph node dissection by video-assisted thoracic surgery for lung cancer. Surg Endosc. 1997;11:703-6.

18. Sagawa M, Sato M, Sakurada A, Matsumura, Endo C, Handa M, et al. A prospective trial of systematic nodal dissection for lung cancer by videoassisted thoracic surgery: can it be perfect? Ann Thorac Surg. 2002;73: 900-4.

19. Kaseda S, Aoki T, Hangai N, Shimizu K. Better pulmonary function and prognosis with video-assisted thoracic surgery than with thoracotomy. Ann Thorac Surg. 2000;70:1644-6.

20. Mountain CF. Revisions in the International System for Staging Lung Cancer. Chest. 1997;111:1710-7.

21. Martini N, Bains MS, Burt ME, Zakowski MF, McCormack P, Rusch VW, et al. Incidence of local recurrence and second primary tumors in resected stage I lung cancer. J Thorac Cardiovasc Surg. 1995;109:120-9.

22. Craig SR, Leaver HA, Yap PL, Pugh GC, Walker WS. Acute phase responses following minimal access and conventional thoracic surgery. Eur J Cardiothorac Surg. 2001;20:455-63.

23. Demmy TL, Plante AJ, Nwogu CE, Takita H, Anderson TM. Discharge independence with minimally invasive lobectomy. Am J Surg. 2004; 188:698-702.

24. Peterson RP, Pham D, Burfiend WR, Hanish SI, Toloza EM, Harpole DH Jr, et al. Thoracoscopic lobectomy facilitates the delivery of chemotherapy after resection for lung cancer. Ann Thorac Surg. 2007;83:1245-9.

25. Scagliotti GV, Fossati R, Torri V, Crino L, Giaccone G, Silvano G, et al. Randomized study of adjuvant chemotherapy for completely resected stage I, II, or IIIA non-small-cell lung cancer. J Natl Cancer Inst. 2003;95:1453-61. 